\title{
Waring-Goldbach Problem: Two Squares and Three Biquadrates
}

\author{
Yingchun Cai* and Li Zhu
}

\begin{abstract}
Assume that $\psi$ is a function of positive variable $t$, monotonically increasing to infinity and $0<\psi(t) \ll \log t /(\log \log t)$. Let $\mathcal{R}_{3}(n)$ denote the number of representations of the integer $n$ as sums of two squares and three biquadrates of primes and we write $\mathcal{E}_{3}(N)$ for the number of integers $n$ satisfying $n \leq N, n \equiv 5,53,101(\bmod 120)$ and

$$
\left|\mathcal{R}_{3}(n)-\frac{\Gamma^{2}(1 / 2) \Gamma^{3}(1 / 4)}{\Gamma(7 / 4)} \frac{\mathfrak{S}_{3}(n) n^{3 / 4}}{\log ^{5} n}\right| \geq \frac{n^{3 / 4}}{\psi(n) \log ^{5} n},
$$

where $0<\mathfrak{S}_{3}(n) \ll 1$ is the singular series. In this paper, we prove

$$
\mathcal{E}_{3}(N) \ll N^{23 / 48+\varepsilon} \psi^{2}(N)
$$

for any $\varepsilon>0$. This result constitutes a refinement upon that of Friedlander and Wooley 2].
\end{abstract}

\section{Introduction}

The celebrated Waring problem involving two squares still remains one of the most elegant problems in additive number theory. Here we outline several pieces of research about it.

Let $v(n)$ denote the number of representations of $n$ as sums of two squares and three nonnegative cubes. In 1972, Linnik 10] proved that $v(n) \gg_{\varepsilon} n^{2 / 3-\varepsilon}$ for all large integers $n$ and any $\varepsilon>0$. In 1981, Hooley [5] improved upon the work of Linnik by obtaining the expected asymptotic formula for $v(n)$. In 2000, he [6] also obtained the asymptotic formula for the number of representations of $n$ as sums of three squares and a $k$-th power.

Let $R_{s}(n)$ denote the number of representations of natural number $n$ as sums of two squares and $s$ biquadrates. The expected asymptotic formula for $R_{s}(n)$ can be established for $s \geq 5$, see Hooley [4]. But for $s \leq 4$, all techniques fail to obtain the expected asymptotic formula for $R_{s}(n)$. Let $E_{s}(N)$ be the number of integers $n \leq N$ such that the expected asymptotic formula for $R_{s}(n)$ fails to be valid. In 2014, Friedlander and Wooley 2] showed

$$
E_{3}(N) \ll N^{1 / 2+\varepsilon} \quad \text { and } \quad E_{4}(N) \ll N^{1 / 4+\varepsilon} .
$$

Received March 28, 2018; Accepted November 14, 2018.

Communicated by Yu-Ru Liu.

2010 Mathematics Subject Classification. 11P32, 11N36.

Key words and phrases. Waring-Goldbach problem, Hardy-Littlewood method, asymptotic formula. This work was supported by The National Natural Science Foundation of China (grant no. 11771333). *Corresponding author. 
Later on, Zhao [13] strengthened these results by showing

$$
E_{3}(N) \ll N^{3 / 8+\varepsilon} \text { and } E_{4}(N) \ll N^{1 / 8+\varepsilon} \text {. }
$$

Let

$$
\Omega=\{n \in \mathbb{N}: n \equiv 5,53,101 \quad(\bmod 120)\} .
$$

The purpose of this paper is to investigate the cognate problem concerning the representation of integers $n$ in $\Omega$ such that

$$
n=p_{1}^{2}+p_{2}^{2}+p_{3}^{4}+p_{4}^{4}+p_{5}^{4},
$$

where $p_{i}$ are prime numbers. The congruence condition is necessary here, since we have $p^{2} \equiv 1$ or $49(\bmod 120)$ and $p^{4} \equiv 1(\bmod 120)$ for primes $p>5$. Denote by $\mathcal{R}_{3}(n)$ the number of representations of natural number $n \in \Omega$ as the form (1.1). By applying a pruning process into the Hardy-Littlewood method, we obtain the following result, which constitutes an improvement upon that of Friedlander and Wooley [2].

Theorem 1.1. For a function $\psi$ of a positive variable $t$, monotonically increasing to infinity and $0<\psi(t) \ll \log t /(\log \log t)$, let $\mathcal{E}_{3}(N)$ be the number of integers $n \in \Omega$ and $n \leq N$ such that

$$
\left|\mathcal{R}_{3}(n)-\frac{\Gamma^{2}(1 / 2) \Gamma^{3}(1 / 4)}{\Gamma(7 / 4)} \frac{\mathfrak{S}_{3}(n) n^{3 / 4}}{\log ^{5} n}\right| \geq \frac{n^{3 / 4}}{\psi(n) \log ^{5} n}
$$

where

$$
\mathfrak{S}_{3}(n)=\sum_{q=1}^{\infty} \frac{1}{\varphi^{5}(q)} \sum_{a(q) *} S_{2}(q, a)^{2} S_{4}(q, a)^{3} e_{q}(-a n) \quad \text { and } \quad S_{k}(q, a)=\sum_{r(q) *} e\left(\frac{a r^{k}}{q}\right) .
$$

Then for any $\varepsilon>0$, we have

$$
\mathcal{E}_{3}(N) \ll N^{23 / 48+\varepsilon} \psi^{2}(N)
$$

\section{Notations and some preliminary lemmas}

In this paper, $\varepsilon \in\left(0,10^{-100}\right)$ and the value of $\varepsilon$ may change from line to line. Let $N$ denote a sufficiently large positive integer in terms of $\varepsilon$. The constants in $O$-term and $\ll$ symbol depend at most on $\varepsilon$. The letter $p$, with or without subscript, is reserved for a prime number. As usual, $\varphi(n)$ denotes Euler's function. We use $e(\alpha)$ to denote $e^{2 \pi i \alpha}$ and $e_{q}(\alpha)=e(\alpha / q)$. We denote by $\sum_{x(q) *}$ a sum with $x$ running over a reduced system of residues modulo $q$. For a set $\mathcal{F},|\mathcal{F}|$ denotes the cardinality of $\mathcal{F}$. 
Lemma 2.1. Let

$$
g_{k}(\alpha)=\sum_{2 \leq p \leq N^{1 / k}} e\left(\alpha p^{k}\right) .
$$

Then for $\alpha=a / q+\lambda,(a, q)=1, q \leq Q$ and $|\lambda| \leq Q /(q N)$, we have

$$
g_{2}(\alpha) \ll Q^{1 / 2} N^{11 / 40+\varepsilon}+V_{2}(\alpha),
$$

where

$$
V_{2}(\alpha)=\frac{N^{1 / 2} \log ^{c} N}{q^{1 / 2-\varepsilon}(1+N|\lambda|)^{1 / 2}}
$$

and $c>0$ denotes some absolute constant.

Proof. It follows from [9, Theorem 2].

Lemma 2.2. Let

$$
S_{k}(q, a)=\sum_{r(q) *} e\left(\frac{a r^{k}}{q}\right)
$$

Then for $(q, a)=1$, we have

(i) $\left|S_{k}(q, a)\right| \ll q^{1 / 2+\varepsilon}$;

(ii) $\left|S_{k}(p, a)\right| \leq((k, p-1)-1) p^{1 / 2}+1$;

(iii) $S_{k}\left(p^{l}, a\right)=0$ for $l \geq \gamma(p)$, where

$$
\gamma(p)= \begin{cases}\theta+2 & \text { if } p^{\theta} \| k, p \neq 2 \text { or } p=2, \theta=0 \\ \theta+3 & \text { if } p^{\theta} \| k, p=2, \theta>0\end{cases}
$$

Proof. For (i), see 7, Lemma 8.5]. For (ii), see [11, Lemma 4.3]. For (iii), see 7, Lemma 8.3].

Lemma 2.3. Let $2 \leq k_{1} \leq k_{2} \leq \cdots \leq k_{s}$ be natural numbers such that

$$
\sum_{i=j+1}^{s} \frac{1}{k_{i}} \leq \frac{1}{k_{j}}, \quad 1 \leq j \leq s-1 .
$$

Then we have

$$
\int_{0}^{1}\left|\prod_{i=1}^{s} g_{k_{i}}(\alpha)\right|^{2} d \alpha \leq N^{1 / k_{1}+\cdots+1 / k_{s}+\varepsilon} .
$$

Proof. By considering the number of solutions of the underlying equation, Lemma 2.3 follows from [1, Lemma 1]. 
Lemma 2.4. Let $\mathcal{F}(N)$ denote a subset of integers in the interval $(N / 2, N]$ and $Z=$ $|\mathcal{F}(N)|$. Let $\xi: \mathbb{Z} \rightarrow \mathbb{C}$ be a function with $|\xi(n)| \leq 1$ for all $n \in \mathbb{Z}$, and set

$$
K(\alpha)=\sum_{n \in \mathcal{F}(N)} \xi(n) e(-n \alpha) .
$$

Then we have
(i) $\int_{0}^{1}\left|g_{2}(\alpha)^{2} K(\alpha)^{2}\right| d \alpha \ll Z^{2} N^{\varepsilon}+Z N^{1 / 2}$;
(ii) $\int_{0}^{1}\left|g_{2}(\alpha)\right|^{11 / 6}\left|g_{4}(\alpha)^{3} K(\alpha)\right| d \alpha \ll N^{2 / 3+\varepsilon} Z+N^{11 / 12+\varepsilon} Z^{1 / 2}$.

Proof. By $[12$, (2.4)] and the bound $|\xi(n)| \leq 1$, we have

$$
\begin{aligned}
\int_{0}^{1}\left|g_{2}(\alpha)^{2} K(\alpha)^{2}\right| d \alpha & =\sum_{p_{1}, p_{2} \leq N^{1 / 2}} \sum_{\substack{m, n \in \mathcal{F}(N) \\
p_{1}^{2}-p_{2}^{2}=n-m}} \xi(m) \overline{\xi(n)} \\
& \ll \sum_{p_{1}, p_{2} \leq N^{1 / 2}} \sum_{\substack{m, n \in \mathcal{F}(N) \\
p_{1}^{2}-p_{2}^{2}=n-m}} 1 \\
& \ll Z^{2} N^{\varepsilon}+Z N^{1 / 2} .
\end{aligned}
$$

By Hölder's inequality and (i), we have

$$
\begin{aligned}
& \int_{0}^{1}\left|g_{2}(\alpha)\right|^{11 / 6}\left|g_{4}(\alpha)^{3} K(\alpha)\right| d \alpha \\
\ll & \left(\int_{0}^{1}\left|g_{2}(\alpha)^{2} g_{4}(\alpha)^{4}\right| d \alpha\right)^{5 / 12}\left(\int_{0}^{1}\left|g_{4}(\alpha)\right|^{16} d \alpha\right)^{1 / 12}\left(\int_{0}^{1}\left|g_{2}(\alpha)^{2} K(\alpha)^{2}\right| d \alpha\right)^{1 / 2} \\
\ll & N^{5 / 12+\varepsilon} N^{1 / 4+\varepsilon}\left(Z^{2} N^{\varepsilon}+Z N^{1 / 2}\right)^{1 / 2} \\
\ll & N^{2 / 3+\varepsilon} Z+N^{11 / 12+\varepsilon} Z^{1 / 2},
\end{aligned}
$$

where Hua's inequality and Lemma 2.3 are used. This completes the proof.

In order to apply the Hardy-Littlewood method, we first define the Farey dissection. For this purpose, we set

$$
A=10^{100}(1+c), \quad Q_{0}=\log ^{A} N, \quad Q_{1}=N^{1 / 4} \quad \text { and } \quad Q_{2}=N^{3 / 4},
$$

where $c$ is defined by (2.1). For $(a, q)=1,0 \leq a<q$, we put

$$
\begin{array}{rlrl}
\mathfrak{M}_{0}(q, a) & =\left(\frac{a}{q}-\frac{Q_{0}^{A}}{N}, \frac{a}{q}+\frac{Q_{0}^{A}}{N}\right], & \mathfrak{M}(q, a) & =\left(\frac{a}{q}-\frac{1}{q Q_{2}}, \frac{a}{q}+\frac{1}{q Q_{2}}\right], \\
\mathfrak{M}_{0} & =\bigcup_{q \leq Q_{0}^{A}} \bigcup_{\substack{a=1 \\
(a, q)=1}}^{q} \mathfrak{M}_{0}(q, a), & \mathfrak{M}=\bigcup_{q \leq Q_{1}} \bigcup_{\substack{a=1 \\
(a, q)=1}}^{q} \mathfrak{M}(q, a), \\
\mathfrak{I} & =\left(-\frac{1}{Q_{2}}, 1-\frac{1}{Q_{2}}\right], & \mathfrak{m}_{1}=\mathfrak{I} \backslash \mathfrak{M}, \quad \mathfrak{m}_{2}=\mathfrak{M} \backslash \mathfrak{M}_{0}, \quad \mathfrak{m}=\mathfrak{m}_{1} \cup \mathfrak{m}_{2} .
\end{array}
$$


Then we have the Farey dissection

$$
\mathfrak{I}=\mathfrak{M}_{0} \cup \mathfrak{m}
$$

Lemma 2.5. For $\alpha \in \mathfrak{m}_{1}$, we have

$$
\left|g_{2}(\alpha)\right| \ll N^{7 / 16+\varepsilon}
$$

Proof. It follows from [3, Theorem 1].

Lemma 2.6. For $(a, q)=1$, let $\mathfrak{N}_{0}(q, a)=\left(\frac{a}{q}-\frac{1}{q N^{7 / 8}}, \frac{a}{q}+\frac{1}{q N^{7 / 8}}\right]$. Then we have

(i) $\sum_{q \leq Q_{0}} \sum_{\substack{a=-q \\(a, q)=1}}^{2 q} \int_{\mathfrak{N}_{0}(q, a)}\left|V_{2}(\alpha)\right|^{1 / 6} d \alpha \ll N^{-\frac{77}{96}+\varepsilon}$;

(ii) $\sum_{q \leq Q_{0}} \sum_{\substack{a=-q \\(a, q)=1}}^{2 q} \int_{\mathfrak{N}_{0}(q, a)}\left|V_{2}(\alpha)\right|^{2} d \alpha \ll Q_{0}^{3}$,

where $V_{2}(\alpha)$ is defined by (2.1).

Proof. By (2.1), we have

$$
\begin{aligned}
& \sum_{q \leq Q_{0}} \sum_{\substack{a=-q \\
(a, q)=1}}^{2 q} \int_{\mathfrak{N}_{0}(q, a)}\left|V_{2}(\alpha)\right|^{1 / 6} d \alpha \\
& \ll \sum_{q \leq Q_{0}} \sum_{\substack{a=-q \\
(a, q)=1}}^{2 q} q^{\varepsilon} \int_{|\lambda| \leq 1 /\left(q N^{7 / 8}\right)} \frac{N^{1 / 12} \log ^{c / 6} N}{(q+q N|\lambda|)^{1 / 12}} d \lambda \\
& \ll N^{-11 / 12+\varepsilon} \sum_{q \leq Q_{0}} \sum_{\substack{a=-q \\
(a, q)=1}}^{2 q} q^{-1 / 12+\varepsilon} \int_{|u| \leq N^{1 / 8} / q} \frac{1}{(1+u)^{1 / 12}} d u \\
& \ll N^{-11 / 12+\varepsilon} Q_{0}^{23 / 12+\varepsilon} \int_{0}^{N^{1 / 8}} \frac{1}{(1+u)^{1 / 12}} d u \ll N^{-77 / 96+\varepsilon} .
\end{aligned}
$$

Now, (i) is proved, and (ii) can be proved by similar arguments.

Lemma 2.7. Let

$$
v_{k}(\lambda)=\sum_{2<n \leq N} \frac{e(n \lambda)}{n^{1-1 / k} \log n} .
$$

Then for $\alpha=a / q+\lambda \in \mathfrak{M}_{0}$, we have

$$
g_{k}(\alpha)=\frac{S_{k}(q, a)}{\varphi(q)} v_{k}(\lambda)+O\left(N^{1 / k} \exp \left(-\log ^{1 / 3} N\right)\right) .
$$


Proof. See [7, Lemma 7.15].

Lemma 2.8. Let $\Omega=\{n \in \mathbb{N}: n \equiv 5,53,101(\bmod 120)\}$, and let

$$
A_{3}(q, n)=\frac{1}{\varphi^{5}(q)} \sum_{a(q) *} S_{2}(q, a)^{2} S_{4}(q, a)^{3} e_{q}(-a n) \quad \text { and } \quad \mathfrak{S}_{3}(n)=\sum_{q=1}^{\infty} A_{3}(q, n) .
$$

Then the series $\mathfrak{S}_{3}(n)$ is convergent and $\mathfrak{S}_{3}(n)>0$ for $n \in \Omega$.

Proof. The convergence of $\mathfrak{S}_{3}(n)$ follows from Lemma 2.2(i). By Lemma 2.2(iii) and the fact that $A_{3}(q, n)$ is multiplicative in $q$, we get

$$
\mathfrak{S}_{3}(n)=\left(1+A_{3}(2, n)+A_{3}(4, n)+A_{3}(8, n)\right) \prod_{p>2}\left(1+A_{3}(p, n)\right) .
$$

When $p>22$, we conclude from Lemma 2.2(ii) that

$$
\left|A_{3}(p, n)\right| \leq \frac{\left(p^{1 / 2}+1\right)^{2}\left(3 p^{1 / 2}+1\right)^{3}}{(p-1)^{4}} \leq \frac{100}{p^{3 / 2}}
$$

So we get

$$
\prod_{p>22}\left(1+A_{3}(p, n)\right) \geq \prod_{p>22}\left(1-\frac{100}{p^{3 / 2}}\right)>c>0 .
$$

Let $L(q, n)$ denote the number of solutions to the congruence

$$
x_{1}^{2}+x_{2}^{2}+x_{3}^{4}+x_{4}^{4}+x_{5}^{4} \equiv n \quad(\bmod q), \quad 1 \leq x_{i} \leq q,\left(x_{i}, q\right)=1 .
$$

Then by [7, Lemma 8.6], we have

$$
\begin{gathered}
1+A_{3}(2, n)+A_{3}(4, n)+A_{3}(8, n)=\frac{L(8, n)}{2^{7}}, \\
1+A_{3}(p, n)=\frac{p L(p, n)}{(p-1)^{5}}
\end{gathered}
$$

For $n \equiv 5,53,101(\bmod 120)$, it is easy to verify that

$$
L(8, n)>0 \quad \text { and } \quad L(p, n)>0 \quad \text { for } 2<p \leq 19 .
$$

Now the conclusion $\mathfrak{S}_{3}(n)>0$ follows from $2.3-2.7$.

3. Mean value estimates

Let

$$
I_{j}=\int_{\mathfrak{m}_{j}}\left|g_{2}(\alpha)^{2} g_{4}(\alpha)^{3} K(\alpha)\right| d \alpha, \quad j=1,2,
$$

where $K(\alpha)$ is defined as in Lemma 2.4. 
Proposition 3.1. We have

$$
I_{1} \ll N^{71 / 96+\varepsilon} Z+N^{95 / 96+\varepsilon} Z^{1 / 2},
$$

where $Z$ is defined as in Lemma 2.4 .

Proof. By Lemma 2.4(ii) and Lemma 2.5, we have

$$
\begin{aligned}
I_{1} & \ll \sup _{\alpha \in \mathfrak{m}_{1}}\left|g_{2}(\alpha)\right|^{1 / 6} \int_{0}^{1}\left|g_{2}(\alpha)\right|^{11 / 6}\left|g_{4}(\alpha)^{3} K(\alpha)\right| d \alpha \\
& \ll N^{7 / 96+\varepsilon}\left(N^{2 / 3+\varepsilon} Z+N^{11 / 12+\varepsilon} Z^{1 / 2}\right) \\
& \ll N^{71 / 96+\varepsilon} Z+N^{95 / 96+\varepsilon} Z^{1 / 2} .
\end{aligned}
$$

Proposition 3.2. We have

$$
I_{2} \ll N^{3 / 4} Q_{0}^{-A / 4} Z+N^{95 / 96+\varepsilon} Z^{1 / 2} .
$$

Proof. For $\alpha \in \mathfrak{m}_{2}$, it follows from Lemma 2.1 with $Q=N^{1 / 4}$ that

$$
\left|g_{2}(\alpha)\right| \ll V_{2}(\alpha)+N^{2 / 5+\varepsilon},
$$

where $V_{2}(\alpha)$ is defined by (2.1). By (3.1) and Lemma 2.4(ii), we get

$$
\begin{aligned}
I_{2} & \ll \int_{\mathfrak{m}_{2}}\left|V_{2}(\alpha)\right|^{1 / 6}\left|g_{2}(\alpha)\right|^{11 / 6}\left|g_{4}(\alpha)^{3} K(\alpha)\right| d \alpha \\
& +N^{1 / 15+\varepsilon} \int_{0}^{1}\left|g_{2}(\alpha)\right|^{11 / 6}\left|g_{4}(\alpha)^{3} K(\alpha)\right| d \alpha \\
& \ll \int_{\mathfrak{m}_{2}}\left|V_{2}(\alpha)\right|^{1 / 6}\left|g_{2}(\alpha)\right|^{11 / 6}\left|g_{4}(\alpha)^{3} K(\alpha)\right| d \alpha+N^{11 / 15+\varepsilon} Z+N^{59 / 60+\varepsilon} Z^{1 / 2} .
\end{aligned}
$$

Let

$$
\mathfrak{N}_{0}(q, a)=\left(\frac{a}{q}-\frac{1}{q N^{7 / 8}}, \frac{a}{q}+\frac{1}{q N^{7 / 8}}\right], \quad \mathfrak{N}(q, a)=\left(\frac{a}{q}-\frac{1}{q Q_{0}}, \frac{a}{q}+\frac{1}{q Q_{0}}\right]
$$

and

$$
\mathfrak{N}_{1}(q, a)=\mathfrak{N}(q, a) \backslash \mathfrak{N}_{0}(q, a) .
$$

From Dirichlet's approximation theorem, we have

$$
\begin{aligned}
& \int_{\mathfrak{m}_{2}}\left|V_{2}(\alpha)\right|^{1 / 6}\left|g_{2}(\alpha)\right|^{11 / 6}\left|g_{4}(\alpha)^{3} K(\alpha)\right| d \alpha \\
\leq & \sum_{q \leq Q_{0}} \sum_{\substack{a=-q \\
(a, q)=1}}^{2 q} \int_{\mathfrak{m}_{2} \cap \mathfrak{N}_{1}(q, a)}\left|V_{2}(\alpha)\right|^{1 / 6}\left|g_{2}(\alpha)\right|^{11 / 6}\left|g_{4}(\alpha)^{3} K(\alpha)\right| d \alpha \\
& +\sum_{q \leq Q_{0}} \sum_{\substack{a=-q \\
(a, q)=1}}^{2 q} \int_{\mathfrak{m}_{2} \cap \mathfrak{N}_{0}(q, a)}\left|V_{2}(\alpha)\right|^{1 / 6}\left|g_{2}(\alpha)\right|^{11 / 6}\left|g_{4}(\alpha)^{3} K(\alpha)\right| d \alpha .
\end{aligned}
$$


For $\alpha=a / q+\lambda \in \mathfrak{N}_{1}(q, a)$, it is easy to see that $q(1+N|\lambda|) \gg N^{1 / 8}$, hence

$$
\sup _{\alpha \in \mathfrak{N}_{1}(q, a)}\left|V_{2}(\alpha)\right| \ll N^{7 / 16+\varepsilon} .
$$

By (3.4), we obtain

$$
\begin{aligned}
& \sum_{q \leq Q_{0}} \sum_{\substack{a=-q \\
(a, q)=1}}^{2 q} \int_{\mathfrak{m}_{2} \cap \mathfrak{N}_{1}(q, a)}\left|V_{2}(\alpha)\right|^{1 / 6}\left|g_{2}(\alpha)\right|^{11 / 6}\left|g_{4}(\alpha)^{3} K(\alpha)\right| d \alpha \\
\ll & \sup _{\alpha \in \mathfrak{N}_{1}(q, a)}\left|V_{2}(\alpha)\right|^{1 / 6} \int_{0}^{1}\left|g_{2}(\alpha)\right|^{11 / 6}\left|g_{4}(\alpha)^{3} K(\alpha)\right| d \alpha \\
\ll & N^{7 / 96+\varepsilon}\left(N^{2 / 3+\varepsilon} Z+N^{11 / 12+\varepsilon} Z^{1 / 2}\right) \\
\ll & N^{71 / 96+\varepsilon} Z+N^{95 / 96+\varepsilon} Z^{1 / 2},
\end{aligned}
$$

where Lemma 2.4(ii) is used. For $\alpha \in \mathfrak{m}_{2}$, we have $q+q N|\lambda| \gg Q_{0}^{A}$. Then it follows from [8, Lemma 3.3] that

$$
\sup _{\alpha \in \mathfrak{m}_{2}}\left|g_{4}(\alpha)\right| \ll N^{31 / 128+\varepsilon}+\frac{N^{1 / 4} \log ^{4} N}{q^{1 / 8-\varepsilon}(1+N|\lambda|)^{1 / 8}} \ll \frac{N^{1 / 4}}{Q_{0}^{A / 9}} .
$$

Moreover for $\alpha \in \mathfrak{N}_{0}(q, a)$, by Lemma 2.1 with $Q=N^{1 / 8}$, we have

$$
\left|g_{2}(\alpha)\right| \ll V_{2}(\alpha)+N^{27 / 80+\varepsilon} .
$$

From (3.6), 3.7) and Lemma 2.6(i)(ii), we get

$$
\begin{aligned}
& \sum_{q \leq Q_{0}} \sum_{\substack{a=-q \\
(a, q)=1}}^{2 q} \int_{\mathfrak{m}_{2} \cap \mathfrak{N}_{0}(q, a)}\left|V_{2}(\alpha)\right|^{1 / 6}\left|g_{2}(\alpha)\right|^{11 / 6}\left|g_{4}(\alpha)^{3} K(\alpha)\right| d \alpha \\
& \ll \sum_{q \leq Q_{0}} \sum_{\substack{a=-q \\
(a, q)=1}}^{2 q} \int_{\mathfrak{m}_{2} \cap \mathfrak{N}_{0}(q, a)}\left|V_{2}(\alpha)^{2} g_{4}(\alpha)^{3} K(\alpha)\right| d \alpha \\
& +N^{99 / 160+\varepsilon} \sum_{q \leq Q_{0}} \sum_{\substack{a=-q \\
(a, q)=1}}^{2 q} \int_{\mathfrak{m}_{2} \cap \mathfrak{N}_{0}(q, a)}\left|V_{2}(\alpha)\right|^{1 / 6}\left|g_{4}(\alpha)^{3} K(\alpha)\right| d \alpha \\
& \ll Z \sup _{\alpha \in \mathfrak{m}_{2}}\left|g_{4}(\alpha)\right|^{3} \sum_{q \leq Q_{0}} \sum_{\substack{a=-q \\
(a, q)=1}}^{2 q} \int_{\mathfrak{N}_{0}(q, a)}\left|V_{2}(\alpha)^{2}\right| d \alpha \\
& +Z N^{99 / 160+\varepsilon} \sup _{\alpha \in \mathfrak{m}_{2}}\left|g_{4}(\alpha)\right|^{3} \sum_{q \leq Q_{0}} \sum_{\substack{a=-q \\
(a, q)=1}}^{2 q} \int_{\mathfrak{N}_{0}(q, a)}\left|V_{2}(\alpha)\right|^{1 / 6} d \alpha \\
& \ll Z N^{3 / 4} Q_{0}^{-3 A / 8} Q_{0}^{3}+Z N^{99 / 160+\varepsilon} N^{3 / 4} Q_{0}^{-A / 2} N^{-77 / 96+\varepsilon} \\
& \ll N^{3 / 4} Q_{0}^{-A / 4} Z \text {, }
\end{aligned}
$$


where the trivial bound $|K(\alpha)| \leq Z$ is used. Now from $(3.2),(3.3),(3.5)$ and $(3.8)$, we get

$$
I_{2} \ll N^{3 / 4} Q_{0}^{-A / 4} Z+N^{95 / 96+\varepsilon} Z^{1 / 2} .
$$

Proposition 3.3. For $N / 2 \leq n \leq N$, we have

$$
\int_{\mathfrak{M}_{0}} g_{2}(\alpha)^{2} g_{4}(\alpha)^{3} e(-n \alpha) d \alpha=\frac{\Gamma^{2}(1 / 2) \Gamma^{3}(1 / 4)}{\Gamma(7 / 4)} \frac{\mathfrak{S}_{3}(n) n^{3 / 4}}{\log ^{5} n}+O\left(\frac{n^{3 / 4} \log \log n}{\log ^{6} n}\right) .
$$

Proof. For $\alpha=a / q+\lambda$, let $f_{k}(\alpha)=\frac{S_{k}(q, a)}{\varphi(q)} v_{k}(\lambda)$. Then it follows from Lemma 2.7 that

$$
\begin{aligned}
& \int_{\mathfrak{M}_{0}} g_{2}(\alpha)^{2} g_{4}(\alpha)^{3} e(-n \alpha) d \alpha \\
= & \int_{\mathfrak{M}_{0}} f_{2}(\alpha)^{2} f_{4}(\alpha)^{3} e(-n \alpha) d \alpha+O\left(n^{3 / 4} \exp \left(-\log ^{1 / 4} n\right)\right) .
\end{aligned}
$$

It is easy to see that

$$
\int_{\mathfrak{M}_{0}} f_{2}(\alpha)^{2} f_{4}(\alpha)^{3} e(-n \alpha) d \alpha=\sum_{q \leq Q_{0}^{A}} A_{3}(q, n) \int_{|\lambda| \leq Q_{0}^{A} / N} v_{2}(\lambda)^{2} v_{4}(\lambda)^{3} e(-n \lambda) d \lambda .
$$

It follows from [7, Lemma 7.16] that

$$
\begin{aligned}
& \int_{|\lambda| \leq Q_{0}^{A} / N} v_{2}(\lambda)^{2} v_{4}(\lambda)^{3} e(-n \lambda) d \lambda \\
= & \int_{0}^{1} v_{2}(\lambda)^{2} v_{4}(\lambda)^{3} e(-n \lambda) d \lambda+O\left(\int_{Q_{0}^{A} / N}^{1} \frac{1}{\lambda^{7 / 4} \log ^{5} N} d \lambda\right) \\
= & \int_{0}^{1} v_{2}(\lambda)^{2} v_{4}(\lambda)^{3} e(-n \lambda) d \lambda+O\left(N^{3 / 4} Q_{0}^{-3 A / 4}\right) .
\end{aligned}
$$

Similar to [7, Lemma 7.19], we have

$$
\int_{0}^{1} v_{2}(\lambda)^{2} v_{4}(\lambda)^{3} e(-n \lambda) d \lambda=\frac{\Gamma^{2}(1 / 2) \Gamma^{3}(1 / 4)}{\Gamma(7 / 4)} \frac{n^{3 / 4}}{\log ^{5} n}+O\left(\frac{n^{3 / 4} \log \log n}{\log ^{6} n}\right) .
$$

By 3.11 and (3.12), we have

$$
\int_{|\lambda| \leq Q_{0}^{A} / N} v_{2}(\lambda)^{2} v_{4}(\lambda)^{3} e(-n \lambda) d \lambda=\frac{\Gamma^{2}(1 / 2) \Gamma^{3}(1 / 4)}{\Gamma(7 / 4)} \frac{n^{3 / 4}}{\log ^{5} n}+O\left(\frac{n^{3 / 4} \log \log n}{\log ^{6} n}\right)
$$

From Lemma 2.2(i) and the inequality $\varphi(q) \gg q / \log q$, we get

$$
\sum_{q \leq Q_{0}^{A}} A_{3}(q, n)=\mathfrak{S}_{3}(n)+O\left(\sum_{q>Q_{0}^{A}} q^{-3 / 2+\varepsilon}\right)=\mathfrak{S}_{3}(n)+O\left(Q_{0}^{-A / 2+\varepsilon}\right) .
$$

Now combining (3.9), (3.10), (3.13) and (3.14), we have

$$
\int_{\mathfrak{M}_{0}} g_{2}(\alpha)^{2} g_{4}(\alpha)^{3} e(-n \alpha) d \alpha=\frac{\Gamma^{2}(1 / 2) \Gamma^{3}(1 / 4)}{\Gamma(7 / 4)} \frac{\mathfrak{S}_{3}(n) n^{3 / 4}}{\log ^{5} n}+O\left(\frac{n^{3 / 4} \log \log n}{\log ^{6} n}\right) \text {. }
$$




\section{Proof of Theorem 1.1}

By the Farey dissection 2.2 , we have

$$
\mathcal{R}_{3}(n)=\int_{\mathfrak{M}_{0}} g_{2}(\alpha)^{2} g_{4}(\alpha)^{3} e(-n \alpha) d \alpha+\int_{\mathfrak{m}} g_{2}(\alpha)^{2} g_{4}(\alpha)^{3} e(-n \alpha) d \alpha .
$$

Let $\psi$ be a function of positive variable $t$, monotonically increasing to infinity and $0<$ $\psi(t) \ll \log t /(\log \log t)$. By Proposition 3.3 and Lemma 2.8 , we may define $\mathcal{F}(N)$ to be the set of integers $n \in \Omega, N / 2 \leq n \leq N$ such that

$$
\left|\mathcal{R}_{3}(n)-\frac{\Gamma^{2}(1 / 2) \Gamma^{3}(1 / 4)}{\Gamma(7 / 4)} \frac{\mathfrak{S}_{3}(n) n^{3 / 4}}{\log ^{5} n}\right| \geq \frac{n^{3 / 4}}{\psi(n) \log ^{5} n} .
$$

For $n \in \mathcal{F}(N)$, by 4.1), 4.2 and Proposition 3.3, we get

$$
\left|\int_{\mathfrak{m}} g_{2}(\alpha)^{2} g_{4}(\alpha)^{3} e(-n \alpha) d \alpha\right| \geq \frac{n^{3 / 4}}{\psi(n) \log ^{5} n} .
$$

For $n \in \mathcal{F}(N)$, let $\xi(n)$ be defined by the following equation

$$
\left|\int_{\mathfrak{m}} g_{2}(\alpha)^{2} g_{4}(\alpha)^{3} e(-n \alpha) d \alpha\right|=\xi(n) \int_{\mathfrak{m}} g_{2}(\alpha)^{2} g_{4}(\alpha)^{3} e(-n \alpha) d \alpha .
$$

Then it is easy to see that $|\xi(n)| \leq 1$. Write $Z(N)=|\mathcal{F}(N)|$. From (4.3), 4.4), we have

$$
\begin{aligned}
\frac{Z(N) N^{3 / 4}}{\psi(N) \log ^{5} N} & \ll \sum_{n \in \mathcal{F}(N)} \frac{n^{3 / 4}}{\psi(n) \log ^{5} n} \\
& \ll \int_{\mathfrak{m}}\left|g_{2}(\alpha)^{2} g_{4}(\alpha)^{3} K(\alpha)\right| d \alpha \\
& \ll\left(\int_{\mathfrak{m}_{1}}+\int_{\mathfrak{m}_{2}}\right)\left|g_{2}(\alpha)^{2} g_{4}(\alpha)^{3} K(\alpha)\right| d \alpha,
\end{aligned}
$$

where

$$
K(\alpha)=\sum_{n \in \mathcal{F}(N)} \xi(n) e(-n \alpha) .
$$

From 4.5, Propositions 3.1 and 3.2, we obtain

$$
\frac{Z(N) N^{3 / 4}}{\psi(N) \log ^{5} N} \ll N^{3 / 4} Q_{0}^{-A / 4} Z(N)+N^{95 / 96+\varepsilon} Z(N)^{1 / 2} .
$$

It follows from (4.6) that

$$
Z(N) \ll N^{23 / 48+\varepsilon} \psi^{2}(N)
$$

Now by (4.7), we have

$$
\mathcal{E}_{3}(N) \ll N^{1 / 3}+\sum_{1 \leq 2^{j} \leq N^{2 / 3}} Z\left(\frac{N}{2^{j}}\right) \ll N^{23 / 48+\varepsilon} \psi^{2}(N),
$$

and the proof of the Theorem 1.1 is completed. 


\section{References}

[1] J. Brüdern, Sums of squares and higher powers I, J. London Math. Soc. (2) 35 (1987), no. $2,233-243$.

[2] J. B. Friedlander and T. D. Wooley, On Waring's problem: two squares and three biquadrates, Mathematika 60 (2014), no. 1, 153-165.

[3] G. Harman, Trigonometric sums over primes I, Mathematika 28 (1981), no. 2, 249254.

[4] C. Hooley, On a new approach to various problems of Waring's type, in: Recent Progress in Analytic Number Theory I, (Durham, 1979), 127-191, Academic Press, London, 1981.

[5] _ On Waring's problem for two squares and three cubes, J. Reine Angew. Math. 328 (1981), 161-207.

[6] _ On Waring's problem for three squares and an $\ell$ th power, Asian J. Math. 4 (2000), no. 4, 885-903.

[7] L. K. Hua, Additive Theory of Prime Numbers, American Mathematical Society, Providence, R.I., 1965.

[8] K. Kawada and T. D. Wooley, On the Waring-Goldbach problem for fourth and fifth powers, Proc. London Math. Soc. (3) 83 (2001), no. 1, 1-50.

[9] A. V. Kumchev, On Weyl sums over primes and almost primes, Michigan Math. J. 54 (2006), no. 2, 243-268.

[10] Y. V. Linnik, Additive problems involving squares, cubes and almost primes, Acta Arith 21 (1972), 413-422.

[11] R. C. Vaughan, The Hardy-Littlewood Method, Second edition, Cambridge Tracts in Mathematics 125, Cambridge University Press, Cambridge, 1997.

[12] T. D. Wooley, Slim exceptional sets for sums of four squares, Proc. London Math. Soc. (3) 85 (2002), no. 1, 1-21.

[13] L. Zhao, Exceptional sets in Waring's problem: two squares and s biquadrates, Acta Arith 162 (2014), no. 4, 369-379.

Yingchun Cai and Li Zhu

School of Mathematical Science, Tongji University, Shanghai, 200092, P. R. China

E-mail address: yingchuncai@mail.tongji.edu.cn, zhuli15@tongji.edu.cn 